\title{
A Study of the CST Parameterization Characteristics
}

\author{
Marco Ceze* \\ University of Michigan, Ann Arbor, MI, 48105, United States \\ Marcelo Hayashi ${ }^{\dagger}$ and Ernani Volpe ‡ $^{\ddagger}$ \\ University of São Paulo, São Paulo, SP, 05508-970, Brazil
}

\begin{abstract}
This paper presents a study of the recently introduced Class-Shape function Transformation (CST). The study assesses some of the main characteristics that parameterization exhibits, such as the sensitivity to parameter perturbations, the uniqueness of a geometric representation, its filtering capability and parameter inter-dependence. In a gradient-based shape optimization process, the solutions of intermediate design cycles are naturally subject to numerical inaccuracy. Under such circumstances, those characteristics of the CST may affect the path and, eventually, compromise the performance of the optimization process depending on the gradient calculation method and minimum-search algorithm. A comparison of two different gradient calculation methods is presented in the context of inverse aerodynamic design along with some validation test cases.
\end{abstract}

\section{Introduction}

A constant endeavor in aerodynamic design is to find the shape that yields optimum performance, according to some context dependent measure of merit. In this process, the geometry parameterization ability to represent context-relevant geometric characteristics is of highly important to the success of the design process. These characteristics are not known a priori, and the way to guarantee the coverage of the entire space of possible geometries is to use each point of the surface as a design variable. This is due to the fact that, in a strict sense, the space of possible geometries has infinite dimension. From the practical point of view, however, such a punctual parameterization presents two essential disadvantages, the large number of parameters (infinite in the limit of geometric representation) and the occurrence of undesired oscillations due to a high number of degrees of freedom.

In this scenario, the need of a higher level parameterization is apparent. This implies in the use of functions to represent the geometry by coefficients that represent the degrees of freedom in the parametric system. Thus, there is a reduction of the space of possible geometries when this space is generated by a base with a finite number of functions. The coefficients that weight these functions can be interpreted as coordinates of a vector $\mathbf{b}$ that assigns a geometry through the function $\mathcal{F}=\mathcal{F}(\mathbf{b})$.

Suppose $\mathcal{F}$ represents an aerodynamic surface that has a round leading edge and a sharp trailing edge (e.g. an airfoil). This implies the function has to be continuous but non-analytic because of the infinite slope at the leading edge and large curvature variations.

A parameterization which has been used widely in aerodynamic optimization works is the Hicks-Henne shape functions $\left({ }^{1-4}\right)$. These functions are added to a baseline geometry to describe geometric modifications. Recently, Kulfan and Bussoletti ${ }^{5}$ proposed a parameterization that is based on Class and Shape functions (CST), which does not require a baseline geometry. This geometric representation has the advantage of directly controlling key engineering parameters, namely, the leading edge radius, the boat-tail angle and the trailing edge thickness. Since it has been introduced, this parameterization has been the subject of a number of studies. ${ }^{4,6-8}$

*Graduate Student, AIAA Member. Email: mceze@umich.edu

†Graduate Student. Email:mhayashi@usp.br

¥Assistant Professor. Email: ernvolpe@usp.br 


\section{CST Parameterization}

The parameterization proposed by Kulfan and Bussoletti ${ }^{5}$ represents a two-dimensional geometry by the product of a class function, $C(x / c)$, and a shape function, $S(x / c)$ plus a term that characterizes the trailing edge thickness:

$$
\frac{y}{c}=C\left(\frac{x}{c}\right) S\left(\frac{x}{c}\right)+\frac{x}{c} \frac{\Delta z_{t e}}{c}
$$

where $C(x / c)$ is given in generic form by:

$$
C\left(\frac{x}{c}\right) \equiv\left(\frac{x}{c}\right)^{N_{1}}\left[1-\frac{x}{c}\right]^{N_{2}} \text { for } 0 \leq \frac{x}{c} \leq 1
$$

The exponents $N_{1}$ and $N_{2}$ define the type of geometry to be represented. An airfoil, for example, is represented by $N_{1}=1 / 2$ and $N_{2}=1$. These values are owed to the fact that the term $\sqrt{x / c}$ enforces a round leading edge, whereas $(1-x / c)$ makes the trailing edge sharp. These features are imposed on the resulting geometry, independently of the particular form of shape function $S(x / c)$ that is adopted. ${ }^{5}$

It can be shown that, for those values of $N_{1}$ and $N_{2}$, the leading edge radius and the boat-tail angle are directly related to the values of the shape function at the extremes of the interval $[0,1] .{ }^{5}$

$$
S(0)=\sqrt{\frac{2 R_{l e}}{c}} \quad S(1)=\tan \beta+\frac{\Delta z_{t e}}{c} .
$$

In principle, the shape function can be arbitrary. However, it is convenient to choose a family of wellbehaved analytical functions, to generate $S(x / c)$, so that the class function $C(x / c)$, should remain as the only source of non-analyticity of the representation as a whole. ${ }^{5}$ It is worth adding that the actual geometry parameterization is inserted into the design cycle solely through the shape function; The class function fully defines the type of geometry the CST represents- e.g. an airfoil contour implies $N_{1}=1 / 2$ and $N_{2}=1$. In most design routines, the geometry type is known a priori therefore, the class function can be assumed fixed within the design cycles.

These characteristics are important, because the shape function acts as a scale function for $C(x / c)$ and, hence, any undesired behavior of $S$ is transferred to the final geometry. In order to illustrate this, one can pick the simplest of shape functions, $S(x / c)=1$, and specify $C(x / c)$ with $N_{1}=1 / 2$ and $N_{2}=1$. As fig. 1 shows, the resulting contour has the basic features of the upper side of an airfoil. Now, on making use of spline control points to parameterize $S(x / c)$, and on perturbing those points, one clearly sees that the spline oscillations are transferred to the final geometry, albeit attenuated.

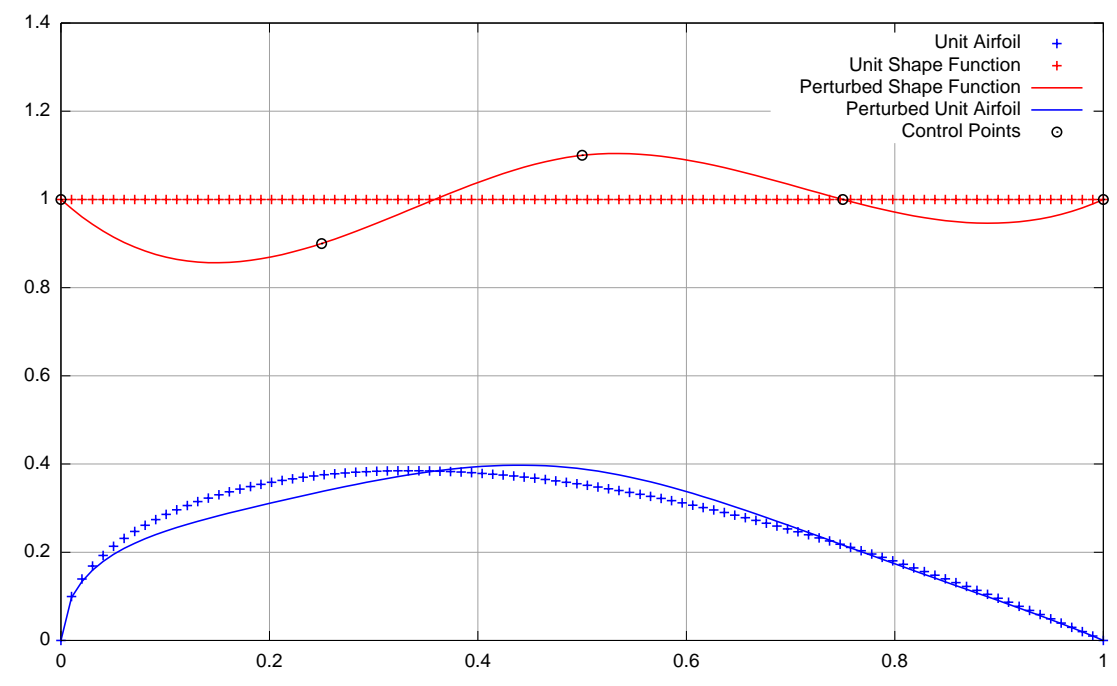

Figure 1. Perturbation of the unit scale function through spline control points.

Kulfan and Bussoletti ${ }^{5}$ have proposed the use of a weighted sum of Bernstein binomials, to represent the shape function $S(x / c)$. These binomials have some remarkable properties, which make them specially suited 
for that role. First, any finite, unweighted summation of them over the closed interval $[0,1]$ yields a unitary result.

$$
B_{p n}\left(\frac{x}{c}\right)=\sum_{i=0}^{n}\left[K_{i, n} \cdot\left(\frac{x}{c}\right)^{i} \cdot\left(1-\frac{x}{c}\right)^{n-i}\right]=1 ; \quad K_{i, n}=\frac{n !}{i !(n-i) !} .
$$

The above summation defines the so-called Bernstein polynomials of $n$th order. Moreover, the binomials have only two real roots, 0 and 1 . All Bernstein binomials are positive within the interval $[0,1]$ in which they present a single maximum. Each of these extrema are located in equally distributed points along that interval. For all these reasons a space of Bernstein polynomials, as defined over the interval $[0,1]$ can be very useful in constructing a parameterization scheme. However, it has a liability in that it lacks an orthogonal basis within that interval which, in least-squares approximation problems, complicates the assembly of convergent sequences of approximation terms. ${ }^{9}$

The shape function, as proposed by Kulfan and Bussoletti, ${ }^{5}$ is defined on the basis of these Bernstein binomials, by the introduction of weight factors $b_{i}$ as follows:

$$
S\left(\frac{x}{c}\right) \equiv \sum_{i=0}^{n}\left[b_{i} \cdot K_{i, n} \cdot\left(\frac{x}{c}\right)^{i} \cdot\left(1-\frac{x}{c}\right)^{n-i}\right] .
$$

the weight factors, in turn, represent the design parameters as introduced previously. In essence, then, $B_{p n}(x / c)$ in Eqn. (4) can be seen as the unitary shape function $S(x / c)=1$, which corresponds to ascribing unity to all weight factors, $b_{i}=1$. Figure 2 depicts the unitary shape function along with its constituent binomials.

On specifying $b_{i} \neq 1$, one can create different shape functions originated by the unitary shape function. Then, an instructive experiment is be to make use of Kulfan and Bussoletti scheme to perturb the unitary

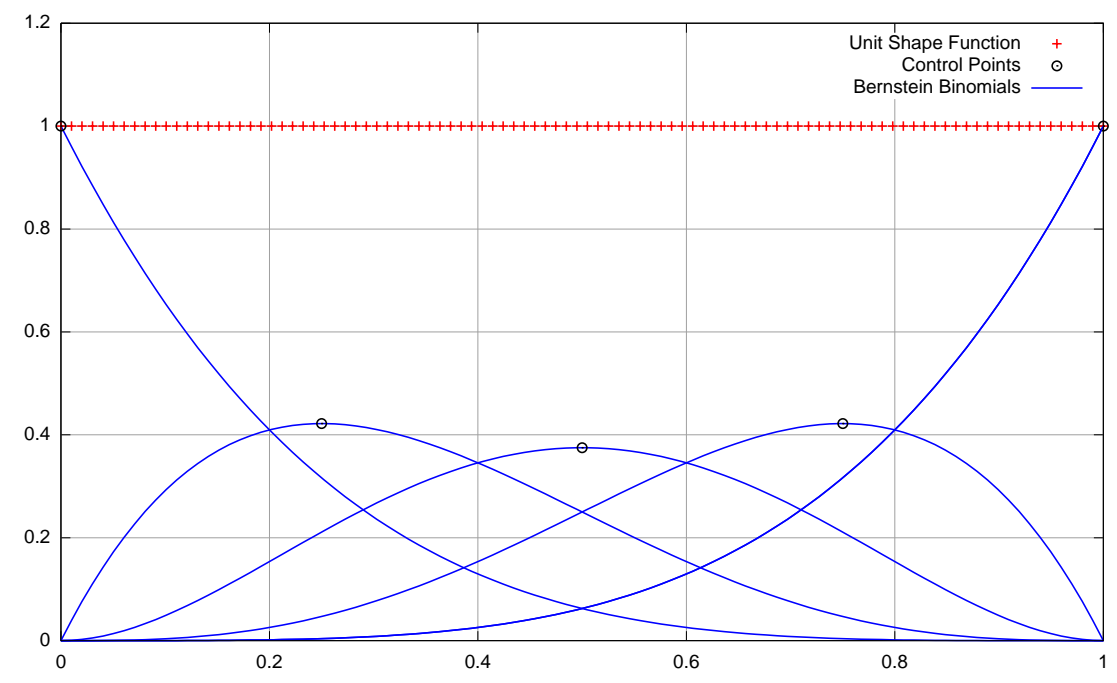

Figure 2. Unit shape function constructed by a $4^{\text {th }}$ order Bernstein polynomial.

function $S(x / c)$, but to do so with precisely the same intensity as was done in the spline case (Fig. 1). On doing so, one gets the result that is depicted in Figure 3, where it can be seen that the final geometry is smoother than that of the previous case.

This experiment illustrates another desirable feature for a geometric parameterization. That is, the above scheme exhibits relatively low sensitivity to abrupt oscillations in the design parameters. Therefore, it displays some filtering capability, which proves useful in shape optimization loops. Moreover, by using analytic functions to construct $S(x / c)$, the scheme has yet another important property, which is to ensure the resulting contours are continuous and differentiable to at least second order. ${ }^{5}$ However, there are some further aspects of the scheme that require careful consideration, in particular with respect to its filtering capability and error distribution. These topics are discussed in the following sections. 


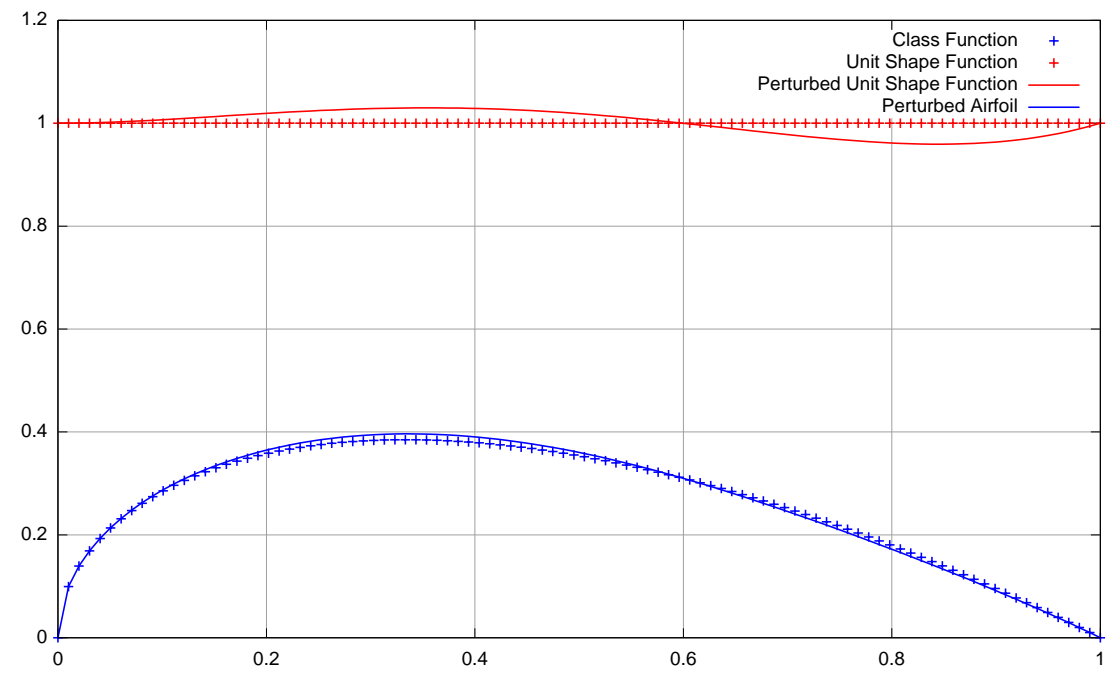

Figure 3. Unit shape function perturbed by Bernstein binomials control parameters.

\section{Airfoil Parameterization}

As was shown above, to parameterize an airfoil, one must first set the class function parameters $N_{1}$ and $N_{2}$ at $1 / 2$ and 1 , respectively. Then, one must pick the order of the parameterization, which fixes the highest order binomial and the number of design parameters. Finally, one has to adjust the shape function parameters, $b_{i}$, so as to fit a particular contour. The latter task can be accomplished by means of the least square error method, where the error is measured by the $L_{2}$ norm of the difference between the CST result and the original geometry.

Figure 4(a) presents the CST fitting to a typical transonic airfoil contour, namely, the RAE2822, along with the original geometry. Figure 4(b) brings the error of such fitting as a function of the order of the parameterization. The error profiles show similar behavior for other airfoils.

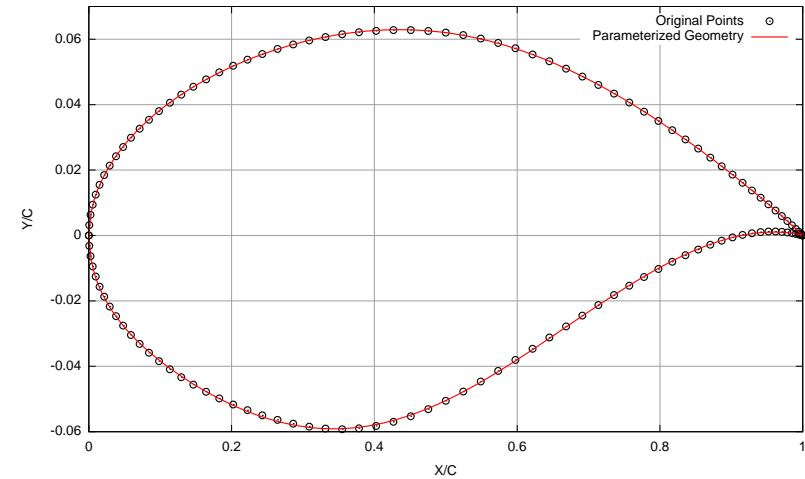

(a) airfoil parameterized by 6 design variables

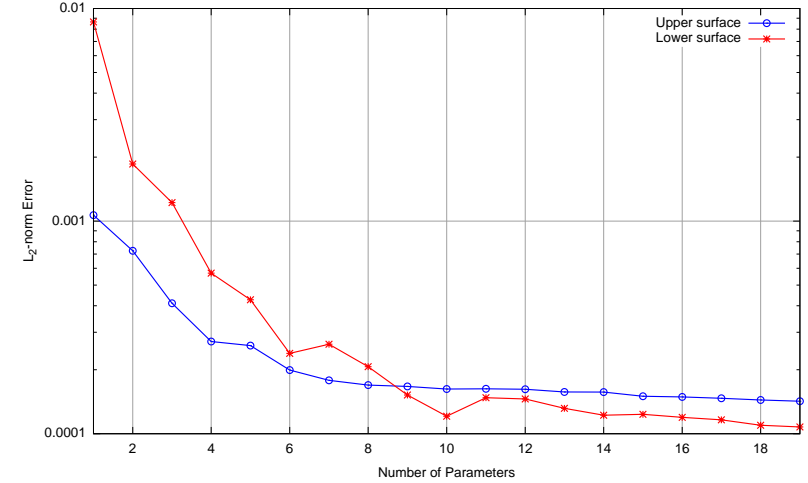

(b) $L_{2}$-norm geometric error

Figure 4. Geometric representation error analysis

\section{III.A. Numerical Uniqueness of the CST Parameterization}

In the context of aerodynamic optimization, it is crucial to check whether the set of design parameters for a given contour is unique within a certain tolerance. That amounts to verifying whether on perturbing those parameters within a predefined level of accuracy, the resulting contour variations are still kept within given bounds. The lack of such property in the parameters space may well imply that the optimization surface is multi-modal, which can clearly degrade the performance of any gradient-based optimization procedure. 
To analyze the uniqueness of the CST parameterization, one can build a set of equations that relates geometry control points $\left[x_{i}, y\left(x_{i}\right)\right]$ directly to design parameters $b_{i}$ :

$$
\underbrace{\left[\begin{array}{ccccc}
S_{0}\left(x_{0}\right) & S_{1}\left(x_{0}\right) & S_{2}\left(x_{0}\right) & \ldots & S_{n}\left(x_{0}\right) \\
S_{0}\left(x_{1}\right) & S_{1}\left(x_{1}\right) & S_{2}\left(x_{1}\right) & \ldots & S_{n}\left(x_{1}\right) \\
\vdots & & \ddots & & \vdots \\
S_{0}\left(x_{n}\right) & S_{1}\left(x_{n}\right) & S_{2}\left(x_{n}\right) & \ldots & S_{n}\left(x_{n}\right)
\end{array}\right]}_{M} \cdot\left\{\begin{array}{c}
b_{0} \\
b_{1} \\
\vdots \\
b_{n}
\end{array}\right\}=\left\{\begin{array}{c}
y\left(x_{0}\right) \\
y\left(x_{1}\right) \\
\vdots \\
y\left(x_{n}\right)
\end{array}\right\}
$$

where:

$$
S_{i}(x)=C\left(x_{i}\right) \cdot K_{i, n} \cdot(x)^{i} \cdot(1-x)^{n-i} .
$$

It is important to stress that the $n+1$ control points must lie within the open interval $(0,1)$, because the extremes of that interval represent the leading and trailing edges, respectively, and these positions are fixed.

A simple assessment of the CST representation uniqueness is given by the spectral condition number of the matrix $M$ in Eqn. (6), $\mathcal{K}(M)$. It is well-known that large values of the condition number indicate wide eigenvalues spectra, therefore characterize a matrix as ill-conditioned. That is, in a linear system such as Eqn. (6), even small changes in $y$ can give rise to large parametric variations $\delta \mathbf{b}$, and vice-versa. It also means that very similar geometries can be generated by widely different parameter sets. In numerical applications, the differences in the resulting geometry might be close to the precision threshold or under the limit of the geometric representation of the boundaries in a computational mesh. Such behavior can interpreted as non-uniqueness, for all practical purposes.

Our numerical tests of the CST scheme seem to indicate that the conditioning of $M$ worsens noticeably, as the order of the parameterization increases. To illustrate this, various uniformly-spaced grids $x_{i}$ are taken, with distinct numbers of points $n$. Each grid corresponds to a parameterization of a different order, i.e. different numbers of parameters. The coarsest grid involves only 2 points (i.e. 1st order parameterization), while the finest one includes 37 points. The matrix $M$ is then computed for each grid, along with its spectral condition number and the eigenvalues themselves. Figure $5(\mathrm{a})$ presents $\mathcal{K}(M)$ as a function of the order of the parameterization, and Fig. 5(b) depicts the distribution of the eigenvalues magnitude for each grid.

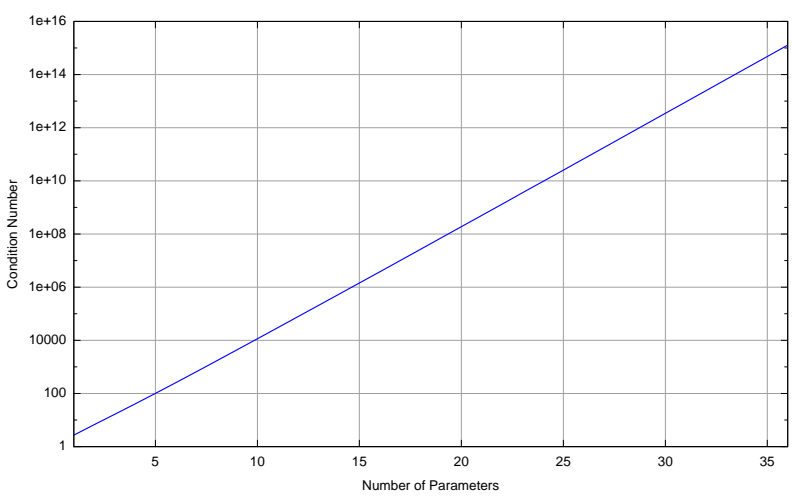

(a) Condition number of the parameterization matrix

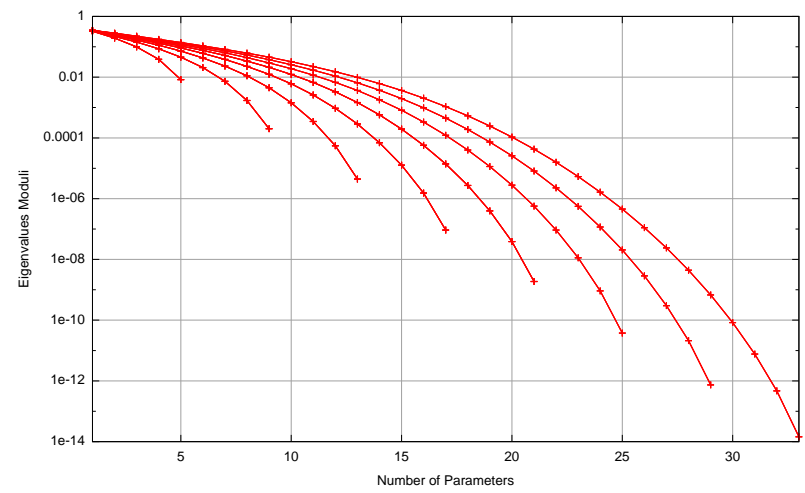

(b) Eigenvalues spectrum

Figure 5. Characteristic-roots analysis of the parameterization matrix.

As can be seen, the conditioning of $M$ degrades markedly as the number of design parameters increases. Moreover, the profiles in Fig. 5(b) show that, although the highest magnitude eigenvalue is precisely the same for all cases, the magnitude of smallest eigenvalues go to zero as the number of parameters grow. That behavior causes the matrix to become virtually singular as the order of the scheme increases.

Other grid distributions of points can also tested and the results for the matrix conditioning are similar. This is mostly due to the combinatory coefficients $K_{i, n}$ in the Bernstein binomials. A thorough geometric study of these binomials can be found in. ${ }^{9}$

In effect, a similar behavior can be noticed when Bernstein binomials are used to construct a Bezier filter for geometric oscillations in inverse aerodynamic design applications, ${ }^{10,11}$ given that this filter makes use of the virtually the same matrix $M$ as the CST scheme. 
A more practical assessment of this characteristic of the CST parameterization can be done through a geometrical representation stand point. That is to find two different sets of parameters $\mathbf{b}$ that represent the same geometry for practical purposes, i.e. negligible geometrical difference. Consider the following system:

$$
B_{i j} \cdot b_{j}=S\left(x_{j}\right) ; \quad B_{i j}=K_{i, n} \cdot\left(x_{j}\right)^{i} \cdot\left(1-x_{j}\right)^{n-i}
$$

where $B_{i j}$ is the Bernstein binomial $i$ applied to point $x_{j}, b_{j}$ is the $j$-th coefficient and $S\left(x_{j}\right)$ is the value of the shape function at $x_{j}$.

As a numerical experiment, one can take the unitary shape function with a high-order parameterization and solve the system (7) for $\mathbf{b}$ and compare the results with the unitary parameter set $b_{j}=1$ which is known a priori to generate $S(x)=1$.

For very high-order parameter sets, the inversion of $B_{i j}$ is not trivial since the matrix becomes close to singular. Therefore, a singular-value-decomposition method is suited for such a task. In this case, the exact inverse of $B_{i j}$ is not achievable or eventually non-existent, instead an approximate inverse, in the least-squares sense, is found. As an example, we present this experiment with a 36 -th order parameterization.

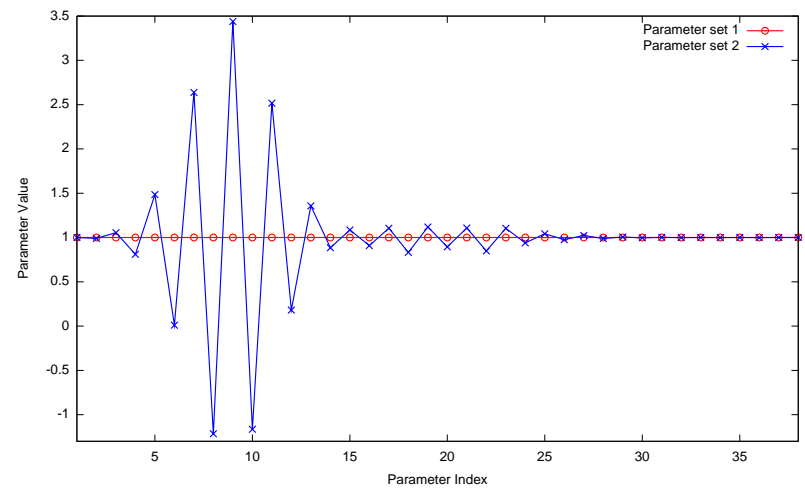

(a) Parameter vectors $\mathbf{b}$

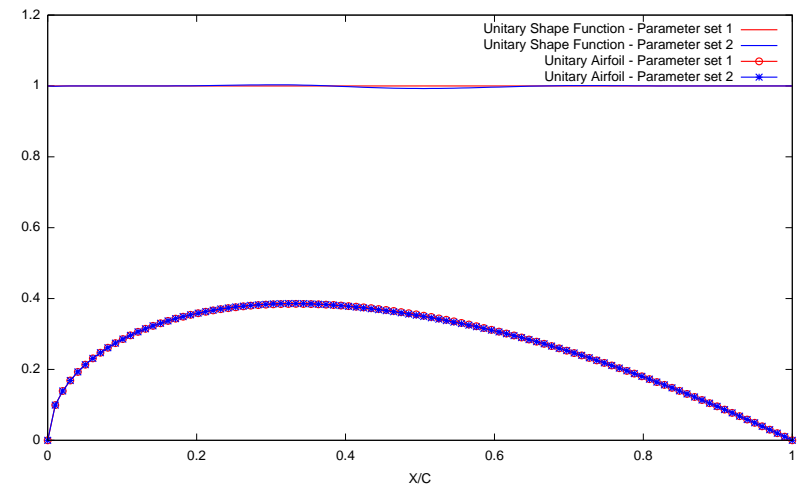

(b) Comparison of the shape functions and corresponding geometries

Figure 6. Assessment of the uniqueness of the parameterization.

Figure 6(a) compares the two parameters set. The set 1 is the unitary vector of parameters. Parameter set 2 is the solution to the system (7) resulted from the method described above. Large variations between both parameter sets are observed, however, the resulting shape functions and geometries do not present significant differences as depicted in Fig. 6(b). In fact, the $L_{2}$-norm of the difference between both shape functions for this case is $7.7545 \cdot 10^{-6}$. This difference is even more attenuated by the application of the airfoil class function, which, as discussed before, damps oscillations in the shape function.

Although this analysis is carried out for airfoil parameterization, the general concept is valid for different classes of geometries, since the shape function is generated independently of the geometry type.

\section{III.B. Parameters study}

A major consequence of the Bernstein binomials properties, as discussed above, is that any parameter change has its effects felt all over the airfoil chord length, to some extent. To explain this, it is convenient to take the unit shape function, again, and analyze the contribution of each binomial to its value. This way, it is possible to assess the influence of a single parameter change along the chord length and, in particular, at the points $x_{i}$ that are the abscissae of the maximum values of the remaining binomials. Therefore these points indicate the position where a change in $b_{i}$ is most prominent and we shall refer to then as control points.

Table 1 lists influence factors for the binomials at each control point for a fourth-order parameterization. These factors represent the contribution of each of the Bernstein binomials to the unitary shape function. The positions $x_{i}$ are the abscissae of the maximum values of the binomials and they correspond to the chord coordinates where a change in $b_{i}$ (Eqn. 5) is most noticeable in the final geometry. Therefore, they can be regarded as control points.

The parameter $b_{0}$, as seen in Table 1 , is the only one that influences its own position, but it also has some effect on the others. The same holds for the last parameter $\left(b_{n}=b_{4}\right)$ with respect to the others. This 
feature can be interpreted as an error distribution, since a local error in one coefficient gets spread along the chord of the airfoil. Besides, it certainly affects the filtering capability the CST has shown to possess.

Table 1. Parameter influence factor ( $4^{t h}$ order).

\begin{tabular}{|c|c|c|c|c|c|}
\hline Binomial & $B\left(x_{0}\right)$ & $B\left(x_{1}\right)$ & $B\left(x_{2}\right)$ & $B\left(x_{3}\right)$ & $B\left(x_{4}\right)$ \\
\hline 0 & 1.00000 & 0.31641 & 0.06250 & 0.00391 & 0.00000 \\
1 & 0.00000 & 0.42188 & 0.25000 & 0.04688 & 0.00000 \\
2 & 0.00000 & 0.21094 & 0.37500 & 0.21094 & 0.00000 \\
3 & 0.00000 & 0.04688 & 0.25000 & 0.42188 & 0.00000 \\
4 & 0.00000 & 0.00391 & 0.06250 & 0.31641 & 1.00000 \\
\hline
\end{tabular}

\section{An Application of the CST Scheme in Aerodynamic Design}

An analysis of the CST scheme in a gradient-based optimization procedure is carried out. The adjoint method is used in the continuous formulation, for 2-D inviscid compressible flow. The objective functional $I$ to be minimized represents the mean square error of the airfoil pressure coefficient $\left(c_{p}\right)$ distribution with respect to a target $c_{p t}$, thus implying an inverse design application. Tests are performed on meshes that are appropriate for the reduced form of the adjoint gradient. ${ }^{12,13}$ The inverse design loop makes use of a simple steepest descent algorithm to evaluate the search direction, and the procedure is carried on until a local minimum is reached within a prescribed tolerance.

\section{IV.A. Gradient Comparison}

In a gradient-based design loop it is important to verify the gradient calculation. A typical approach for this is to compare the adjoint-based gradient with a finite-difference form of the gradient. The finite-difference gradient is calculated by individually perturbing each of the parameters and computing an approximate value for the gradient of the objective functional with respect to a variation of the parameters. In this work, the parameters were perturbed by $\pm 1 \%$ and a second-order centered finite difference scheme was used to computed the components of the finite-difference-gradient. For this approach, the number of flow solutions is twice the number of parameters and each of these solutions is required to comply with a strict convergence criterion of $10^{-10}$ on the flux residuals.

The gradient-comparison case we present here consists of a NACA0012 airfoil as the starting geometry and the target pressure distribution is prescribed from a known geometry, the NACA0009. The flow regime is $M_{\infty}=0.7$ and $0^{\circ}$ of angle of attack. Since both geometries and pressure distributions are symmetric the analysis is focused on the upper portion of the geometry which is represented by 6 parameters.

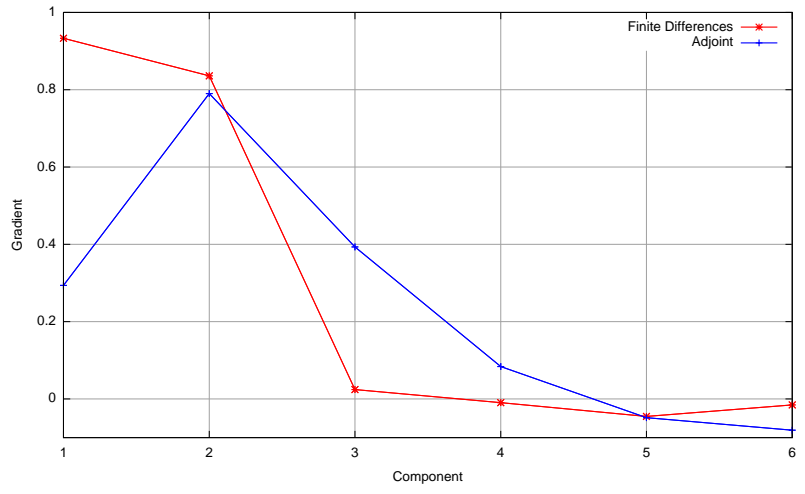

(a) Gradient components

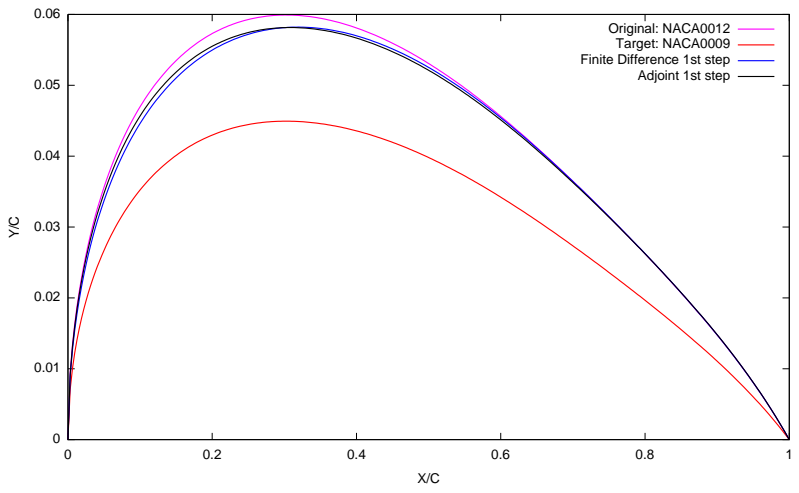

(b) 1-st step geometry comparison

Figure 7. Comparison of gradient calculation methods. 
The initial geometry, NACA0012, is given by the following set of parameters:

$$
\mathbf{b}=[0.170374 ; 0.160207 ; 0.143643 ; 0.166426 ; 0.110476 ; 0.179433] \text {. }
$$

Figure 7(a) shows the components of the gradient for the first step of the design loop. Despite the weak agreement between the gradients calculated by the two methods, the geometries resulted from the first step of the loop are quite similar as shown in Fig. 7(b). The step-size used in the steepest descent method for this case is 0.01 .

\section{IV.B. Validation Cases}

The inverse design loop validation cases consist of choosing as target-pressure-distribution the pressure distribution of a known geometry. This way, it is guaranteed that the desired pressure distribution is achievable. However, there is no guarantee that there is only one geometry capable of producing such a pressure distribution. In this case, a local minimum of the optimization surface is found and therefore it satisfies the initial design premises.

For the validation cases presented here, the target pressure distributions are obtained by numerically solving the inviscid flow equations for geometries that are generated with sets of parameters calculated by least-squares curve-fitting similarly to Fig. 4. This process is required to guarantee that the targetdistribution geometry lies in the space of geometries generated the parameterization method, therefore, it enables, a priori, the full convergence of the design loop.

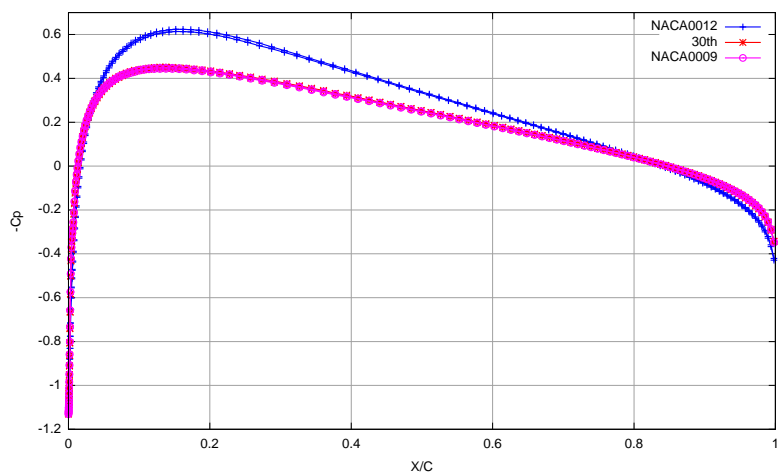

(a) $C_{p}$

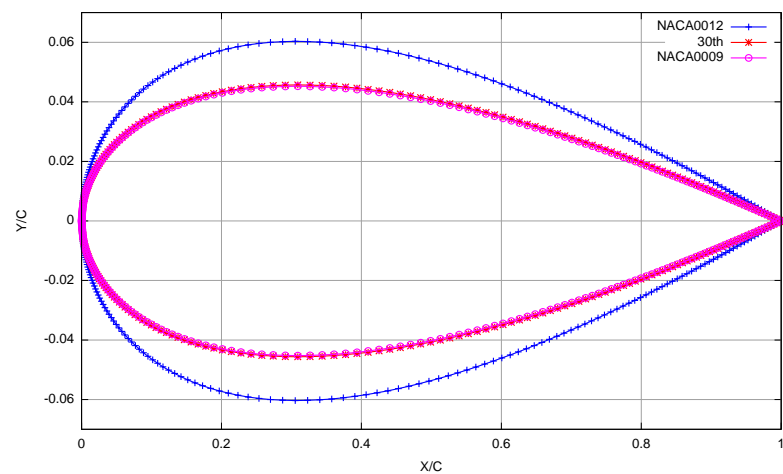

(b) Geometry

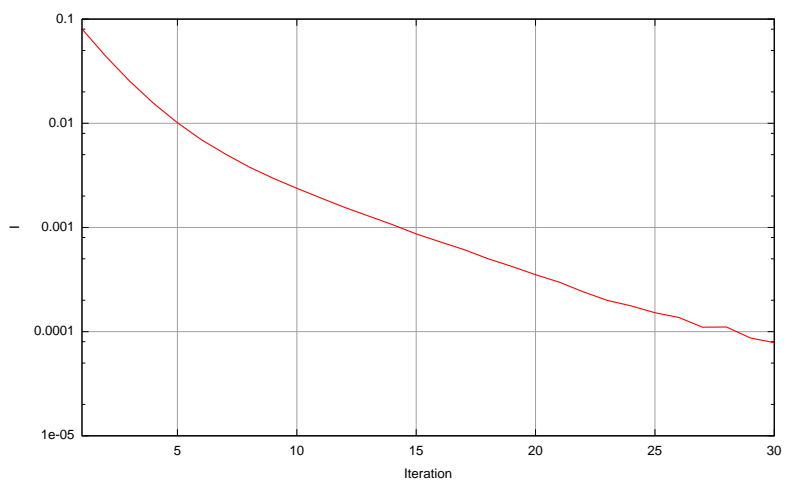

(c) Objective function convergence history

Figure 8. Inverse design test case with 2 parameters $-M_{\infty}=0.7$, AOA $=0^{\circ}$ : NACA0012 to NACA0009.

Figure 8 presents the results of a validation case where the initial geometry is the NACA0012 airfoil and the target-distribution geometry is the NACA0009. The flow regime is $M_{\infty}=0.7, \alpha=0^{\circ}$ and the geometries are generated by sets of 2 parameters, i.e. first-order parameterization. In the design cycle, the step size used in the steepest descent method was 0.01 and, despite the simplicity of this method, the convergence rate of the objective function is satisfactory (Fig. 8(c)). The differences in the pressure distributions of NACA0009 and the 30th are not significant as seen in Fig. 8(a) and the corresponding geometries are also 
very similar to each other (Fig 8(b)). Note that a low order parameterization was used, so that the effect of the conditioning of the matrix $M$ is not expected and the parameter influence factors are minimal.

The second case consists of the same geometries and flow regime as the previous case. However, the geometries are represented by a fifth-order parameterization. The objective of this case is to compare the performance of the design loop when the geometries are represented by parameterizations of different orders. This case was ran with a step size of 0.01 in the steepest descent method according to the previous case. Figures 9 and 9 (b) present the results of the 30th cycle. Figure 9(c) shows the comparison of the loop performance with the geometry represented by 2 and 6 parameters. Note that for both cases, the parameterization order is low enough so the effect of the ill-conditioning of the matrix $M$ can be disregarded.

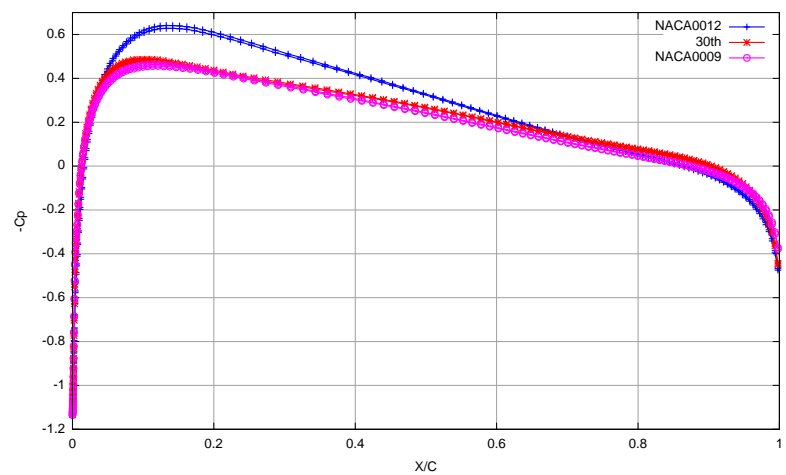

(a) $C_{p}$

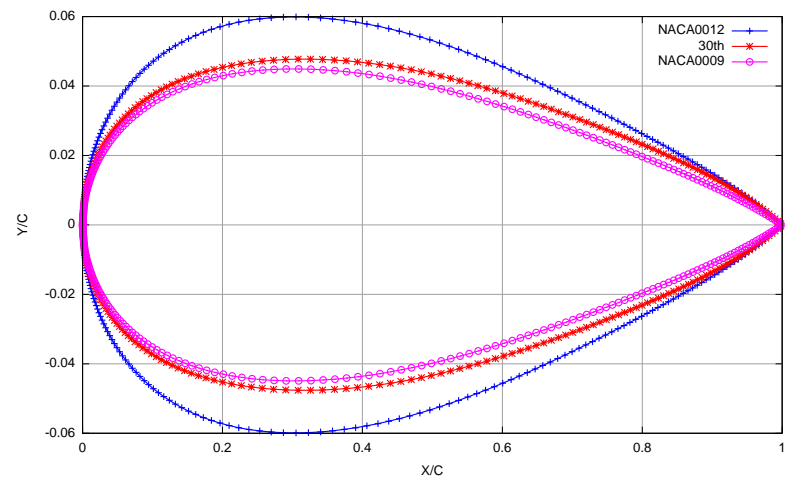

(b) Geometry

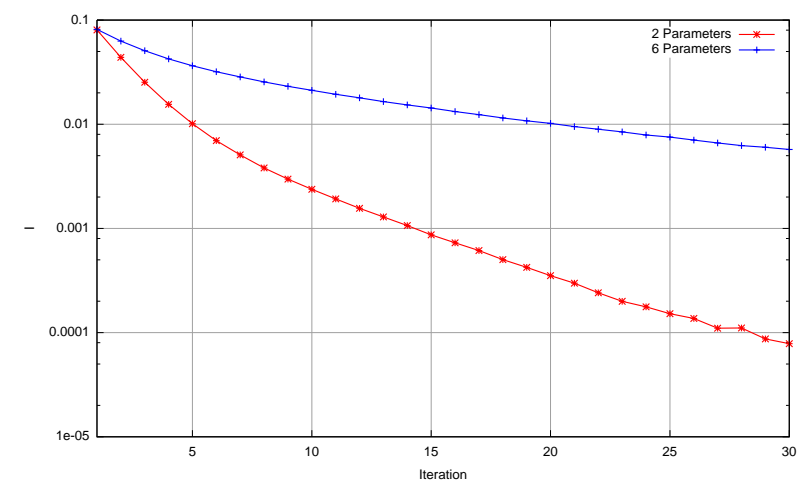

(c) Objective function convergence history comparison

Figure 9. Inverse design test case with 6 parameters and constant step size of $0.01-M_{\infty}=0.7$, AOA $=0^{\circ}:$ NACA0012 to NACA0009.

Similarly to the second case, the third case consists of the NACA0012 as the baseline geometry and NACA0009 as target. The flow regime is the same as the first case but, instead of a constant step size, a sequence of step sizes is used in the steepest descent method. The first 9 iterations used a step size of 0.001 , the 10th through the 21st iterations were ran with 0.01 and the subsequent cycles used a step size of 0.1. Figure 10(c) presents the objective-function convergence history and Figs. 10(a) and 10(b) presents the 30th solution compared to the target. In these figures, it is observed a remaining difference between the target and the 30th pressure distributions in the vicinity of the leading and the trailing edges. Consequently, there is also a remaining difference between the geometries in those regions.

Figure 11 presents a higher-order parameterization case where the original geometry NACA 0012 was modified to achieve the pressure distribution of a RAE 2822 at Mach 0.75 and $1.0^{\circ}$ of angle of attack. Both geometries were generated with 11 parameters and the step size for the steepest descent method is 0.01 . It is worth noting that even with the high condition number of the parameterization, the results show that the method converges almost to the target, matching the shock position and strength. However, there are still remaining differences in the vicinity of the leading and trailing edges which, in this and the previous cases, present an extremely low rate of change. This slow rate is possibly related to the steepest descent method and the high influence factors in those regions indicate an additional cause for these remaining differences. The oscillatory behavior in Fig. 11(c) is mostly related to the shifting position of the shock wave, which, 


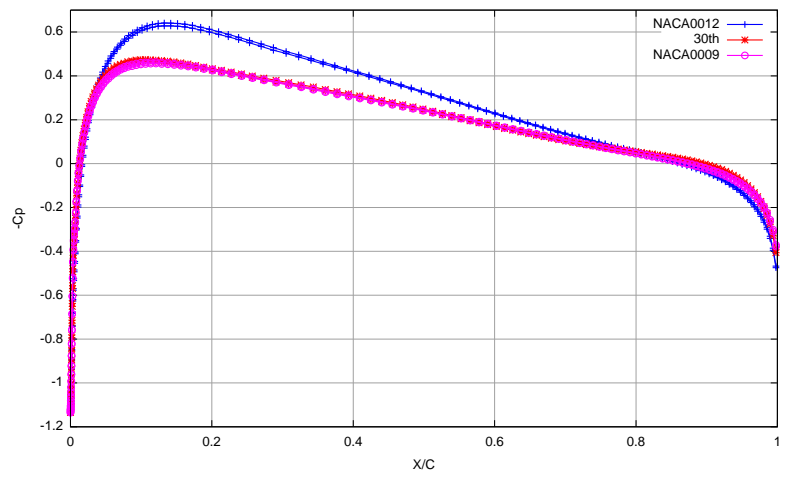

(a) $C_{p}$

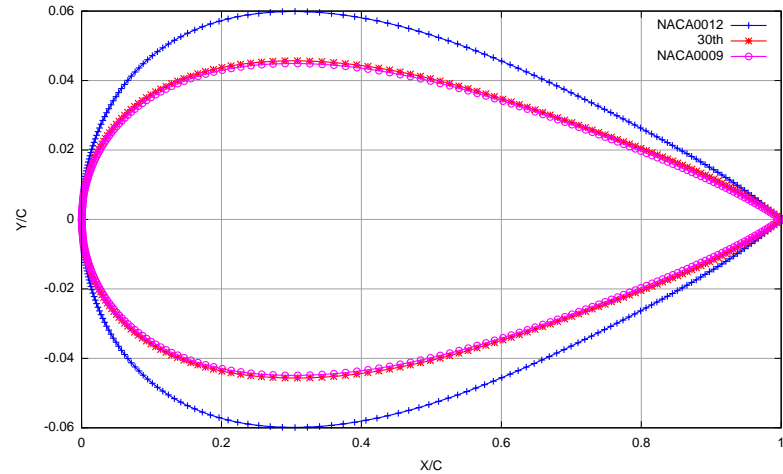

(b) Geometry

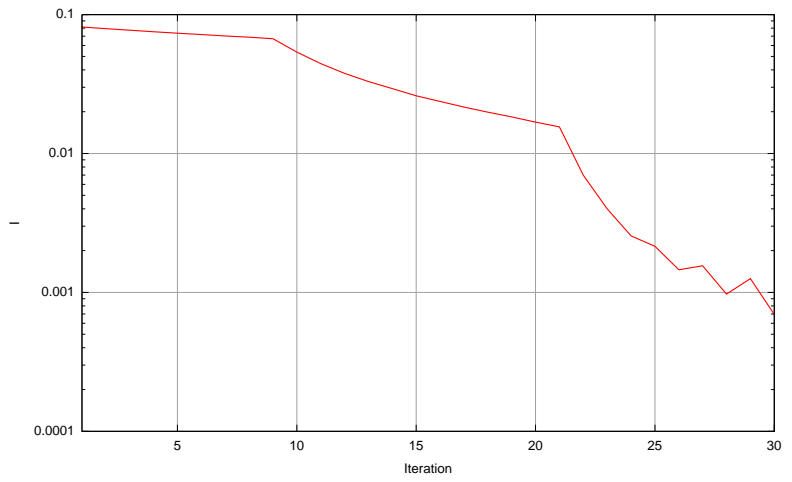

(c) Objective function convergence history

Figure 10. Inverse design test case with 6 parameters and a sequence of step sizes $-M_{\infty}=0.7$, AOA $=0^{\circ}:$ NACA0012 to NACA0009.

when misplaced, contributes significantly to the objective functional.

\section{Conclusions}

The CST parameterization presents desirable characteristics in both aerodynamics and optimization fields. However, it is perceived that some of its properties have yet to be further investigated. This work presents some insight on these aspects and their possible effects on gradient-based optimization methods.

The smooth behavior of Bernstein-based shape functions is a desirable characteristic for aerodynamic shape parameterization in a design cycle since $\mathrm{C} 2$ continuity is readily available from the approach proposed by Kulfan \& Bussoletti. ${ }^{5}$ Additionally, the filtering and error distribution characteristics discard the need for explicit filters in numerical aerodynamic design loops. However, the results and experiments presented in this paper indicate that these features might decrease the convergence rate of the design cycle in regions of the geometry where the contributions of the parameterization terms to the shape function are high. In fact, the comparison presented in Fig. 9(c) shows a faster convergence rate for a lower-order parameterization. The regions where the influence factors are high, namely, the vicinity of the leading and trailing edges, might be affecting the overall convergence of the design loop since those are the regions where there are remaining differences between the pressure distributions of the current geometry and the target geometry.

Experimentation with the CST scheme have shown that the numerical non-uniqueness of the geometric representation is prominent in higher order parameterization. For airfoil representation, the very high order shape functions are not typically necessary, therefore, the ill-conditioning of the parameterization matrix is not relevant to the overall performance of the airfoil design loops. However, for different classes of geometries, the higher order parameterizations may be necessary and non-uniqueness can become an important issue.

Another aspect regarding the ill-conditioning of the matrix $M$ is the susceptibility of the design cycle to numerical errors. The condition number of the parameterization matrix roughly indicates the scaling factor between an error in the geometry and the corresponding error in the parameters. Therefore, numerical errors 


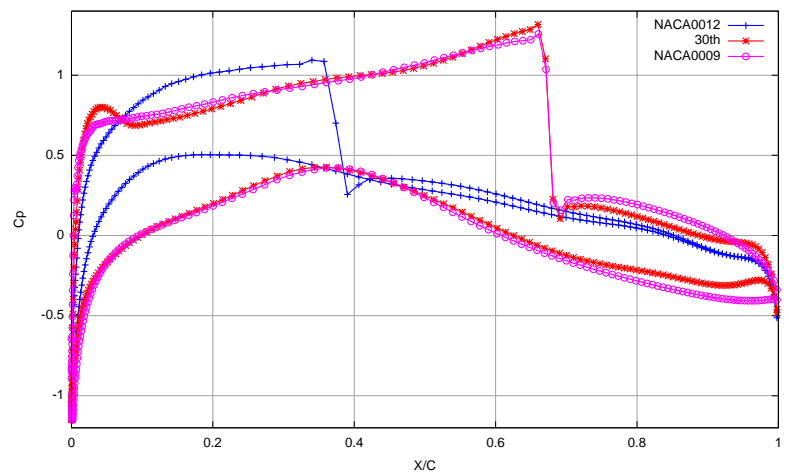

(a) $C_{p}$

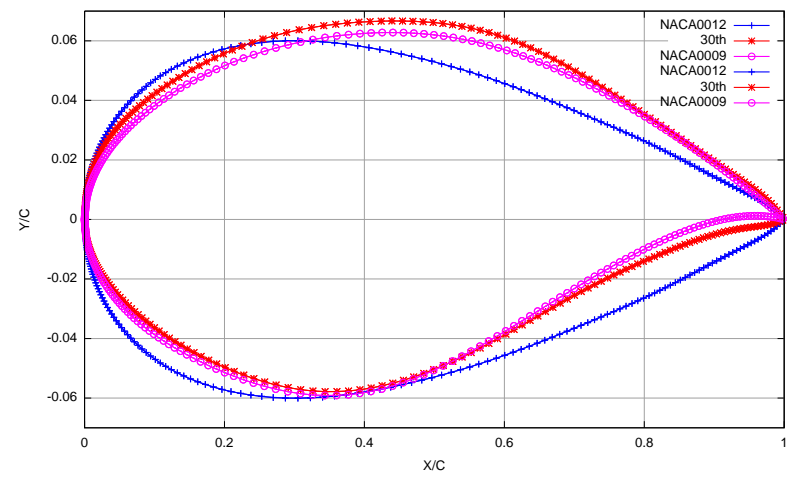

(b) Geometry

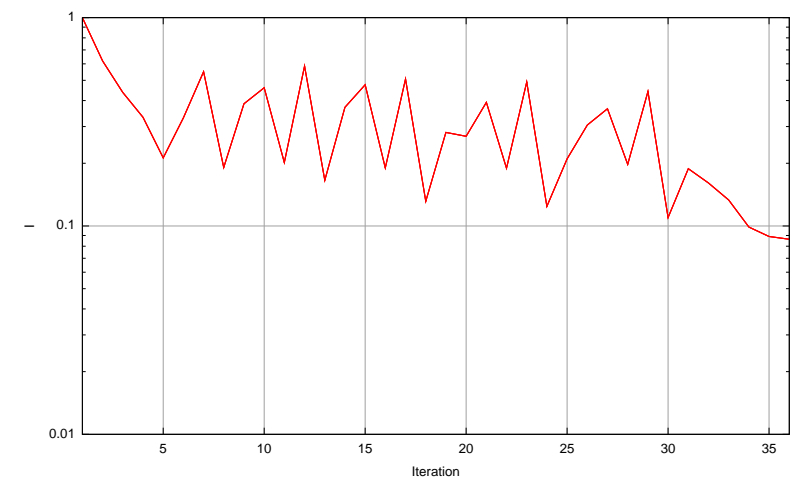

(c) Objective function convergence history

Figure 11. Inverse design test case with 11 parameters $-M_{\infty}=0.75, \mathbf{A O A}=1^{\circ}:$ NACA0012 to RAE2822.

can be amplified in the parametric space.

Finally, the comparison of the gradient calculation methods suggests that a more appropriate approach for validating the gradient of an aerodynamic optimization loop is direct comparison of the resulting geometry, since similar geometries can be represented by widely different parameter sets when using higher order shape functions.

\section{Acknowledgements}

The authors acknowledge the contribution of the NDF laboratory at the University of São Paulo in providing the required resources for the execution of this work. The authors are also grateful for the advice given by Professor Krzysztof J. Fidkowski to this paper. 


\section{References}

${ }^{1}$ Hicks, R. M. and Henne, P. A., "Wing design by numerical optimization," Journal of Aircraft, 1978. 1996.

${ }^{2}$ Reuther, J. J., Aerodynamic Shape Optimization Using Control Theory, Ph.D. thesis, University of California Davis,

${ }^{3}$ Kim, H.-J., Sasaki, D., Shigeru, and Nakahashi, K., "Aerodynamic Optimization of Supersonic Transport Wing Using Unstructured Adjoint Method," AIAA Journal, 2001.

${ }^{4}$ Mousavi, A., Castonguay, P., and Nadarajah, S. K., "Survey of Shape Parameterization Techniques and its Effect on Three-Dimensional Aerodynamic Shape Optimization," AIAA 37th Fluid Dynamics Conference and Exhibit, 2007.

${ }^{5}$ Kulfan, B. M. and Bussoletti, J. E., "Fundamental Parametric Geometry Representations for Aircraft Component Shapes," 11th AIAA/ISSMO Multidisciplinary Analysis and Optimization Conference, 2006.

${ }^{6}$ Kulfan, B. M., "Recent Extensions and Applications of the "CST" Universal Parametric Geometry Representation Method," 7th AIAA Aviation Technology, Integration and Operations Conference, 2007.

${ }^{7}$ Ceze, M. A., Projeto Inverso Aerodinâmico Utilizando o Método Adjunto Aplicado às Equações de Euler, Master's thesis, University of São Paulo, 2008.

${ }^{8}$ Antunes, A. P., Azevedo, J. L. F., and da Silva, R. G., "A Framework for Aerodynamic Optimization Based On Genetic Algorithms," 47th Aerospace Sciences Meeting Including The New Horizons Forum and Aerospace Exposition, No. AIAA 2009-1094, 2009.

${ }^{9}$ Farouki, R. T., "Legendre-Bernstein basis transformations," Journal of Computational and Applied Mathematics, 2000. ${ }^{10}$ Volpe, E. V., "A3 - Inverse Aerodynamic Design Applications," Tech. rep., EMBRAER - FAPESP - EPUSP, 2005.

${ }^{11}$ Volpe, E. V., Oliveira, G. L., Santos, L. C. C., Hayashi, M. T., and Ceze, M. A. B., "Inverse Aerodynamic Design Applications Using The MGM Hybrid Formulation," Inverse Problems in Science and Engineering, Vol. 17, No. 2, March 2009, pp. 245-261.

${ }^{12}$ Jameson, A. and Kim, S., "Reduction of the Adjoint Gradient Formula in the Continuous Limit," 41st Aerospace Sciences Meeting \& Exhibit, 2003.

${ }^{13}$ Jameson, A. and Kim, S., "Reduction of the Adjoint Gradient Formula for Aerodynamic Shape Optimization Problems," AIAA Journal, Vol. 41, No. 11, November 2003, pp. 2114-2129. 